\title{
Zum Rauchverhalten von Studierenden in Österreich
}

\section{- Ein empirischer Befund -}

Schätzungen zufolge sterben in Österreich jährlich ca. 14.000 Personen an den Folgen des Rauchens. Um Diskussionen zum Rauchverhalten führen und gesetzliche Maßnahmen zur Eindämmung des Rauchens zielgerichtet ergreifen bzw. anpassen zu können, erscheint es notwendig, möglichst umfangreiche Informationen über das Rauchverhalten der österreichischen Bevölkerung einzuholen. Dies wird im vorliegenden Beitrag versucht, in welchem das Rauchverhalten von Studierenden in Österreich untersucht wird. Im Zuge dieser Untersuchung konnte festgestellt werden, dass ungefähr ein Drittel der annähernd 2.000 befragten Studierenden raucht. Als häufigste Gründe für das Rauchen werden Gewohnheit und Entspannung genannt. Für Nichtraucher stehen Gesundheits- und Kostenüberlegungen als Gründe für das Nichtrauchen im Vordergrund. Gesetzliche Einschränkungen des Rauchens spielen dagegen kaum eine Rolle für das Nichtrauchen und dürften auch nur wenige ehemalige Raucher zum Nichtrauchen bewogen haben. Derartige Maßnahmen dienen somit in erster Linie dem Schutz von Nichtrauchern.

\section{Gerhard Reichmann, Margit Sommersguter-Reichmann ${ }^{1}$}

\section{Einleitende Bemerkungen}

Unterschiedliche Aspekte des Rauchens waren in den letzten Jahren immer wieder Gegenstand öffentlicher Diskussionen, politischer Bekundungen sowie gesetzlicher Maßnahmen. So gab es zahlreiche Debatten bezüglich der negativen gesundheitlichen Folgen des Rauchens für die aktiven Raucher. Wissenschaftliche Untersuchungen belegen die Gefährlichkeit des Rauchens. ${ }^{2}$ Schätzungen zufolge sterben in Österreich jährlich ca. 14.000 Personen an den Folgen des Rauchens. ${ }^{3}$ Um Raucher von ihrem morbiditäts- und mortalitätsfördernden Verhalten abzubringen, wurden mehrfach Aufklärungskampagnen gestartet, Präventionsmaßnahmen eingeführt ${ }^{4}$ sowie einzelne gesetzliche Vorschriften erlassen. $\mathrm{Zu}$ nennen wären hier vor allem die laut österreichischem Tabakgesetz vorgeschriebenen Warnhinweise auf Zigarettenpackungen ${ }^{5}$, das ebenfalls im Tabakgesetz festgelegte Werbe- und Sponsoringverbot für Tabakerzeugnisse ${ }^{6}$ sowie die in den Jugendschutzgesetzen der Länder normierten Konsumund Verkaufsverbote für Tabakwaren ${ }^{7}$. Ein weiterer Aspekt des Rauchens, der zur Zeit im Mittelpunkt des Interesses steht, ist der Schutz von Nichtrauchern. Immerhin

Gerhard Reichmann, Margit Sommersguter-Reichmann, Universität Graz, Institut für Informationswissenschaft und Wirtschaftsinformatik/Institut für Finanzwirtschaft, Graz gibt es in Österreich jährlich mehr als 1.000 Todesfälle als Folge des Passivrauchens. ${ }^{8}$ Zum Schutz von Nichtrauchern wurden mittlerweile unterschiedliche Maßnahmen gesetzt. So gibt es für einzelne Bereiche gesetzliche Vorschriften: Das österreichische ArbeitnehmerInnenschutzgesetz schreibt etwa einen Schutz von Nichtrauchern vor Tabakrauch am Arbeitsplatz vor ${ }^{9}$, das Tabakgesetz normiert überhaupt ein generelles Rauchverbot in öffentlichen Gebäuden ${ }^{10}$. In anderen Bereichen, wie z.B. der Gastronomie, versucht man, einen gewissen Nichtraucherschutz durch freiwillige Maßnahmen zu erreichen. ${ }^{11}$ Als besonders heikel werden Eingriffe in den privaten Wohnbereich erachtet, die aber zum Schutz von Kindern rauchender Eltern durchaus überlegenswert erscheinen und teilweise auch von Ärzten vehement gefordert werden. ${ }^{12}$ Diesbezüglich gibt es derzeit lediglich Vorüberlegungen hinsichtlich möglicher Maßnahmen.

Um die eben genannten Diskussionen zielgerichtet führen und die daraus folgenden politischen und gesetzlichen Maßnahmen ergreifen und anpassen zu können, erscheint es notwendig, möglichst umfangreiche Informationen über das Rauchverhalten der österreichischen Bevölkerung einzuholen. Dies erweist sich allerdings insofern als problematisch, da es kaum relevante Untersuchungen gibt, auf deren Ergebnisse (Daten) man zugreifen könnte. ${ }^{13}$ Die zentrale einschlägige Datenbasis, nämlich jene der $\mathrm{WHO}^{14}$, weist nur stark aggregierte Informationen zum Rauchverhalten auf, die zudem nicht immer auf dem neuesten Stand sind. ${ }^{15}$ Man kann dieser Datenbank u.a. 
den Raucheranteil der über 15 Jahre alten Bevölkerung, der in Österreich $29 \%{ }^{16}$ beträgt, sowie den jährlichen Zigarettenkonsum pro Person, der in Österreich bei $1.260 \mathrm{Zi}$ garetten ${ }^{17}$ liegt, entnehmen. ${ }^{18}$ Keinerlei Informationen lassen sich dagegen hinsichtlich jenes Alters, in dem im Durchschnitt am häufigsten mit dem Rauchen begonnen wird, gewinnen. Derartige Informationen wären für zielgerichtete Präventionsmaßnahmen äußerst wichtig. Ebenso wenig gibt die Datenbank Aufschlüsse über die Gründe für das Rauchen. Die Kenntnis dieser Gründe wäre für die Ausgestaltung zahlreicher Maßnahmen zur Eindämmung des Rauchens von essentieller Bedeutung. Praktisch keine Daten existieren auch hinsichtlich des Rauchverhaltens spezifischer Bevölkerungsgruppen in Österreich, wie beispielsweise hinsichtlich des Rauchverhaltens von Schülern, Lehrlingen oder Studierenden.

Genau an den eben genannten Informationslücken setzt die gegenständliche Studie an. Sie soll einen ersten, detaillierteren Einblick in das Rauchverhalten einer bestimmten Bevölkerungsgruppe, nämlich jenes der Studierenden, geben. Betrachtet man die bisherigen Publikationen zum Rauchverhalten von Studierenden, so lässt sich erkennen, dass diesbezügliche Untersuchungen vor allem im angloamerikanischen Raum bereits öfters durchgeführt wurden. Loukas/Garcia/Gottlieb (2006) befragten beispielsweise mehr als 1.000 U.S.-amerikanische (texanische) Studierende bezüglich ihres Rauchverhalten sowie hinsichtlich ihrer Meinung zu Anti-Raucher-Maßnahmen. Biasco/Hartnett (2002) haben speziell die Einstellung U.S.-amerikanischer Studierender zum Rauchen anhand einer stichprobenartigen Befragung Studierender der Northwest Florida University analysiert. Emmons u.a. (1998) haben sich vor allem auf die Identifizierung möglicher Einflussfaktoren auf das Rauchverhalten von Studierenden konzentriert. Auch für einzelne andere Staaten existieren bereits einschlägige Studien. ${ }^{19}$ In Österreich wurden dagegen bislang noch keinerlei Untersuchungen zum Rauchverhalten von Studierenden durchgeführt. Insofern stellt die gegenständliche Arbeit eine Pilotstudie dar.

Der Aufbau der Arbeit ist wie folgt: Im 2. Abschnitt werden der Gegenstand der Untersuchung und die geplanten Zielsetzungen nochmals näher erläutert. Zudem wird ein kurzer Überblick über ähnlich ausgerichtete Studien in anderen Staaten gegeben. Der Beschreibung des Erhebungsdesigns bzw. der Erhebung ist der 3. Abschnitt gewidmet. Im vierten Abschnitt werden die Ergebnisse zum Rauchverhalten von Studierenden präsentiert und interpretiert. Den Abschluss bildet ein kurzes Resümee.

\section{Gegenstand der Untersuchung}

Die hier vorgestellte Studie dient einer Erhebung des aktuellen Rauchverhaltens einschließlich der Ursachen für das Rauchen bzw. Nichtrauchen von Studierenden in Österreich („Rauchverhalten“), der Analyse möglicher soziodemografischer und sonstiger Einflussfaktoren auf das Rauchverhalten („Einflussfaktoren“) sowie der Erhebung der Einstellung von Rauchern und Nichtrauchern zum Rauchverhalten („Einstellung“).

Infolge begrenzter Ressourcen wurde die Untersuchung auf Studierende jeweils eines Vertreters jedes in Österreich vorkommenden Universitätstypus beschränkt; d.h. es wurden Studierende einer „klassischen“ (Voll-)Universität, einer Technischen Universität, einer Medizinischen Universität sowie einer Kunstuniversität befragt. Für die Befragung wurden vier (der fünf ${ }^{20}$ ) Universitäten im Bundesland Steiermark herangezogen. Somit sollten die Ergebnisse hinsichtlich des Rauchverhaltens von Studierenden in der Steiermark besonders aussagekräftig sein. Zu betonen ist, dass es sich bei der vorliegenden Untersuchung um eine deskriptive Studie handelt.

\subsection{Rauchverhalten}

Das wichtigste Kriterium im Hinblick auf das Rauchverhalten stellt im Rahmen der gegenständlichen Arbeit das aktuelle Rauchverhalten der Studierenden dar. Hier ist zunächst einmal zwischen Rauchern und Nichtrauchern zu unterscheiden. Es stellt sich die Frage, ob sich das aktuelle Rauchverhalten der Studierenden von jenem der österreichischen Gesamtbevölkerung unterscheidet. Neben den bereits erwähnten WHO-Daten können hier auch die Ergebnisse von Haidinger/Waldhoer/Vutuc (1998) als Vergleichsbasis herangezogen werden. Demnach waren 37\% der im Jahre 1995 repräsentativ befragten Österreicher Raucher und 63\% Nichtraucher. In Abhängigkeit vom Raucherstatus (Raucher/Nichtraucher) sollen in der Folge die Gründe für das jeweilige Verhalten untersucht werden.

\section{a. Raucher}

Innerhalb der Gruppe der Raucher soll erhoben werden, seit wann die Befragten rauchen, um im Hinblick auf allfällige Präventionsmaßnahmen ermitteln zu können, in welchem Alter mit dem Rauchen begonnen wurde. Interessant erscheint die Frage, ob viele Studierende erst während des Studiums mit dem Rauchen beginnen. ${ }^{21}$ Sollte dies der Fall sein, so wären verstärkte Aufklärungs- und Präventionsmaßnahmen für Studienanfänger, eventuell kombiniert mit verschärften Rauchverboten an Universitäten und in Wohneinrichtungen für Studierende überlegenswert. Sollte die Mehrzahl der Studierenden zwischen Vollendung des 16. und 18. Lebensjahres mit dem Rauchen beginnen, wäre dagegen eine Ausweitung des Jugendschutzes auf diese Gruppe in Erwägung zu ziehen. Stellt sich hingegen heraus, dass sehr viele Studierende bereits vor dem vollendeten 16 . Lebensjahr mit dem Rauchen beginnen, wären die derzeitigen Schutzmaßnahmen für diese Gruppe kritisch zu hinterfragen. In diesem Zusammenhang wird gleichzeitig erhoben, wie viele Zigaretten täglich konsumiert werden.

Spricht man mit Rauchern, so äußern diese oftmals den Wunsch, mit dem Rauchen aufzuhören. Zum Teil werden derartige Bemühungen sogar durch Leistungen aus der gesetzlichen Sozialversicherung unterstützt ${ }^{22}$. 
Dieses Änderungspotential hinsichtlich des Raucherstatus soll für rauchende Studierende im Rahmen der gegenständlichen Untersuchung erhoben werden. Anhand der Ergebnisse lässt sich erkennen, welche Maßnahmen zur Senkung der Raucherzahlen verstärkt ergriffen werden sollten. Entweder sind das Maßnahmen, die Raucher mit dem Wunsch zum Aufhören unterstützen, oder Maßnahmen, die bei „überzeugten“ Rauchern diesen Wunsch erst auslösen sollen. ${ }^{23}$

Ein zentraler Untersuchungspunkt für die Gruppe der Raucher stellt die Erhebung der Gründe für das aktuelle Rauchverhalten dar. Kennt man diese Gründe im Detail, lassen sich Aufklärungs- und Präventionsmaßnahmen, aber auch Verbote optimieren. Sollten etwa zahlreiche Studierende angeben, infolge „Gruppenzwangs“ zu rauchen, würde dies für Aufklärungskampagnen sprechen, in denen das Nichtrauchen als positiv und erstrebenswert dargestellt wird. So könnten z.B. erfolgreiche (nichtrauchende) Universitätsabsolventen für das Nichtrauchen „werben“. Sollten dagegen viele Studierende aus Gründen der Stressbewältigung rauchen, müsste Hilfestellung angeboten werden, wie der Stress mit anderen Methoden abzubauen ist.

\section{b. Nichtraucher}

Innerhalb der Gruppe der Nichtraucher soll zwischen ehemaligen Rauchern und Personen, die nie geraucht haben, unterschieden werden. In Analogie zur Gruppe der Raucher sollen die Gründe für das Nichtrauchen erhoben werden, um weitere Anhaltspunkte für geeignete Maßnahmen zur Senkung der Raucherzahlen zu gewinnen. Wenn beispielsweise zahlreiche Studierende angeben sollten, aus Kostengründen nicht (mehr) zu rauchen, würde dies für eine weitere Erhöhung der Preise von Tabakwaren sprechen. Würden hingegen häufig Rauchverbote als Grund genannt, wäre zu überlegen, derartige Verbote noch deutlich weiter auszudehnen.

\subsection{Einflussfaktoren}

Im Zuge dieser Untersuchung soll weiters erhoben werden, ob ein Zusammenhang zwischen aktuellem Rauchverhalten und gewissen soziodemographischen Faktoren bzw. sonstigen Rahmenbedingungen ${ }^{24}$ besteht. Haidinger/Waldhoer/Vutuc (1998) haben u.a. festgestellt, dass monatliches Haushaltseinkommen, Dauer der Schulbildung sowie Größe des Wohnortes (gemessen an der Einwohnerzahl) im Gegensatz zum Alter keinen Einfluss auf das Rauchverhalten ausüben. In verschiedenen Untersuchungen wurden weiters das Geschlecht sowie das Rauchverhalten der Eltern als Einflussfaktoren identifiziert. $^{25}$

In der vorliegenden Studie soll der Einfluss der Faktoren „Universität", „Geschlecht“, „Alter“, „Kinder“, „Studienbeihilfe" und "Rauchverhalten der Eltern" auf das Rauchverhalten der Studierenden untersucht werden. Hinsichtlich des Faktors „Universität“ wird angenom- men, dass es an den Medizinischen Universitäten weniger Raucher als an den übrigen Universitätstypen gibt. Die Vermutung liegt nahe, dass Personen, die künftig in Gesundheitsberufen tätig sein wollen, ein überdurchschnittliches Gesundheitsbewusstsein aufweisen und sich zudem ihrer Vorbildfunktion bewusst sind. ${ }^{26}$ Bezüglich des Faktors „Geschlecht" wird erwartet, dass es weniger rauchende Studentinnen als Studenten gibt, da es auch insgesamt weniger Raucherinnen als Raucher gibt. Allerdings ist in den letzten Jahren eine deutliche Zunahme des Raucheranteils unter Frauen festzustellen, was mittelbis langfristig zu einer Angleichung des Rauchverhaltens von Frauen und Männern führen könnte. ${ }^{27}$ Haidinger/ Waldhoer/Vutuc (1998) haben in ihrer Untersuchung festgestellt, dass Personen „mittleren“ Alters (zwischen 25 und 45 Jahren) am häufigsten rauchen. Dies lässt vermuten, dass Studierende mit zunehmenden Alter eher rauchen. Hingegen wird angenommen, dass Studierende, die bereits Eltern sind, aus Rücksichtnahme auf die Gesundheit ihres Kindes/ihrer Kinder und/oder im Sinne einer Vorbildwirkung eher nicht rauchen. ${ }^{28}$ Ebenso könnte der Anteil der Nichtraucher innerhalb der Gruppe der Studienbeihilfenbezieher höher sein, da Rauchen mittlerweile mit hohen Kosten verbunden ist. Da Untersuchungen gezeigt haben ${ }^{29}$, dass das Rauchverhalten der Eltern einen starken Einfluss auf das Rauchverhalten ihrer Kinder ausübt, wird zudem das aktuelle Rauchverhalten der Eltern erhoben und in der Folge der diesbezügliche Einfluss analysiert.

\subsection{Einstellung}

Ergänzend zum Rauchverhalten und zum möglichen Einfluss soziodemographischer und sonstiger Faktoren auf dieses soll im Zuge der gegenständlichen Studie auch die Einstellung zum Rauchen bzw. Rauchverhalten erhoben werden. In diesem Zusammenhang interessiert insbesondere die Einschätzung der Schädlichkeit des Rauchens sowie des Passivrauchens seitens der Raucher und Nichtraucher. Obwohl es eindeutige Belege für die Schädlichkeit des Zigarettenrauches und damit sowohl des Aktiv- als auch des Passivrauchens gibt ${ }^{30}$, wird diese Tatsache oftmals - in erster Linie von Rauchern - nicht akzeptiert. Weiters soll festgestellt werden, inwieweit Studierende der Meinung sind, dass Raucher zum Nichtrauchen ermuntert werden sollten. Vermutlich gibt es diesbezüglich erhebliche Einstellungsunterschiede zwischen Rauchern und Nichtrauchern.

Im Zuge von Diskussionen über die Notwendigkeit von Rauchverboten an unterschiedlichen Orten, wie etwa in Gastronomiebetrieben, wird häufig - vor allem seitens der Nichtraucher - argumentiert, dass derartige Verbote infolge der Rücksichtslosigkeit von Rauchern gegenüber Nichtrauchern unbedingt notwendig seien, um einen angemessenen Nichtraucherschutz zu gewährleisten. Seitens der Raucher wird den Nichtrauchern in diesem Zusammenhang oftmals mangelnde Toleranz vorgeworfen. Nachfolgend soll überprüft werden, inwieweit auch Studierende 
der Meinung sind, dass sich Raucher tendenziell rücksichtslos gegenüber Nichtrauchern bzw. Nichtraucher tendenziell intolerant gegenüber Rauchern verhalten.

\section{Erhebungsdesign}

Zur Erreichung der oben genannten Zielsetzungen sollten stichprobenartig ausgewählte Studierende anhand eines strukturierten Fragebogens befragt werden. Der Beschreibung des Erhebungsdesigns im Sinne von Fragebogen und Stichprobe ist dieser Abschnitt gewidmet.

\subsection{Fragebogen}

Ausgangspunkt der Datenerhebung war somit die Entwicklung eines Fragebogens, dessen Inhalt in Tabelle 1 dargestellt ist. ${ }^{31}$ Dieser Inhalt lässt sich den in Abschnitt 2 diskutierten drei Themenkomplexen "Rauchverhalten“, „Einflussfaktoren“ und „Einstellung“ zuordnen.

Die zentrale Frage zum Themenkomplex „Rauchverhalten" ist jene nach dem aktuellen Rauchverhalten, die zugleich als Filterfrage dient. Nur Raucher werden in der Folge danach gefragt, seit wie vielen Jahren sie schon rauchen, wie viele Zigaretten sie im Durchschnitt pro Tag konsumieren und ob sie mit dem Rauchen aufhören wollen. Die Fragen nach der Dauer des aktuellen Rauchverhaltens und nach dem täglichen Zigarettenkonsum dienen u.a. als Kontrollfragen hinsichtlich des aktuellen Rauchverhaltens. Weiters werden Raucher nach den Gründen für das Rauchen gefragt, wobei in Anlehnung an die Literatur ${ }^{32}$ im Fragebogen aus den vorgegebenen Gründen „Gruppenzwang bzw. Gruppenzugehörigkeit“, "Gewohnheit“, „Entspannung bzw. Stressbewältigung“, "Zeitvertreib“, „Geschmack“ und „Gewicht" auszuwählen ist. Mehrfachnennungen sind möglich. Ergänzend ist noch die Antwortmöglichkeit "Sonstiges" vorgesehen.

\section{Im Unter-}

schied dazu werden Nichtraucher nur danach gefragt, ob sie schon immer Nichtraucher waren (im Folgenden als „absolute Nichtraucher" bezeichnet) und welche Gründe für ihr aktuelles Rauchverhalten ausschlaggebend sind. Dabei sind

Tabelle 1: Inhalt des Fragebogens

Quelle: eigene Darstellung ebenfalls in Anlehnung an die Literatur ${ }^{33}$ als Gründe "Gesundheit“, „Kosten“, „Gesetzliche Rauchverbote“34, „Warnhinweise "35, „Gesellschaftlicher Trend “36 und „Sonstiges“ als Antwortmöglichkeiten vorgegeben.

Zu den Fragen bezüglich der Einflussfaktoren ist Folgendes anzumerken: Für die Variable „Universität“ sind die vier Ausprägungen „U“ („klassische“ Universität), „TU“ (Technische Universität), „MU“ (Medizinische Universität) und „KU“ (Kunstuniversität) vorgesehen. Die Variable „Alter" weist als einzige dieses Themenkomplexes kardinales Messniveau auf, da nach dem Alter in Jahren gefragt wird. Die Fragen nach Kindern und Studienbeihilfe haben ein nominales Messniveau und weisen jeweils nur zwei Antwortmöglichkeiten auf („,ja“ oder „nein“), sind also dichotom. Ebenso dichotom sind die Fragen nach dem aktuellen Rauchverhalten der Mutter sowie des Vaters (Antwortmöglichkeiten: „Raucher" oder „Nichtraucher“), die im Rahmen der Auswertung zur Variablen „Rauchverhalten der Eltern“ zusammengefasst werden; Voraussetzung für die Zuordnung zur Ausprägung „Nichtraucher" ist dabei, dass beide Elternteile nicht rauchen.

Sowohl Raucher als auch Nichtraucher werden wiederum mit dem dritten Themenkomplex, den fünf Fragen bezüglich der Einstellung zum Rauchen, konfrontiert. Diese Fragen, die als Aussagen formuliert sind, weisen ordinales Messniveau auf, wobei die 5-stufige Antwortskala von starker Zustimmung (++) über geringe Zustimmung $(+)$, Neutralität (neutral) und geringe Ablehnung (-) bis zu starker Ablehnung (-) reicht. Zudem ist jeweils noch die Antwortmöglichkeit „will bzw. kann ich nicht beant-

\begin{tabular}{|llll|}
\hline Themenkomplex & Untersuchungsbereich & Frage & Messniveau \\
\hline Rauchverhalten & Raucher und Nichtraucher & Aktuelles Rauchverhalten & nominal \\
& Raucher & Dauer des aktuellen Rauchverhaltens & kardinal \\
& & Zigarettenkonsum pro Tag & kardinal \\
& & Wille zur Änderung des Rauchverhaltens & nominal \\
& Nichtraucher & Gründe für das aktuelle Rauchverhalten & nominal \\
& & Dauer des aktuellen Rauchverhaltens & nominal \\
& Raucher und Nichtraucher & nominal \\
& & Gründe für das aktuelle Rauchverhalten & nominal \\
& & Geschlecht & nominal \\
& & Alter & kardinal \\
& & Kinder & nominal \\
& & Studienbeihilfe & nominal \\
& & Rauchverhalten der Eltern & nominal \\
& Raucher und Nichtraucher & Schädlichkeit des Rauchens & ordinal \\
& & Schädlichkeit des Passivrauchens & ordinal \\
& & Ermunterung zum Nichtrauchen & ordinal \\
& & Rücksichtslosigkeit von Rauchern & ordinal \\
& & Intoleranz von Nichtrauchern & ordinal \\
\hline
\end{tabular}


worten" vorgesehen. Die konkreten Aussagen lauten: „Ich halte Rauchen für gesundheitsschädlich!“, „Ich halte Passivrauchen für gesundheitsschädlich!“, „Raucher sollten zum Nichtrauchen ermuntert werden!“, „Ich halte Raucher für rücksichtslos gegenüber Nichtrauchern!“ und „Ich halte Nichtraucher für intolerant gegenüber Rauchern!“.

\subsection{Stichprobe}

Im Zuge der Datenerhebung sollte eine Auswahl von Studierenden in Österreich befragt werden. In einem ersten Schritt wurden vier Universitäten ausgesucht, an denen die Befragung durchgeführt werden sollte: Als Repräsentantin für die „klassischen“ Universitäten die Karl-Franzens-Universität Graz (UG), als Repräsentantin für die Technischen Universitäten die Technische Universität Graz (TUG), als Repräsentantin für die Medizinischen Universitäten die Medizinische Universität Graz (MUG) und als Repräsentantin für die Kunstuniversitäten die Kunstuniversität Graz (KUG). Die Einschränkung auf Universitäten in der Steiermark war notwendig, da die Interviews von Studierenden der UG im Rahmen von Lehrveranstaltungen durchgeführt werden sollten. Geplant war, an jeder der ausgewählten Universitäten eine Stichprobe im Umfang von 5\% der Studierenden zu befragen. Für die UG war zusätzlich eine Schichtung nach Fakultäten vorgesehen. Insgesamt sollten somit ${ }^{37} 1.950$ Studierende befragt werden.

Während der Erhebungsphase, die sich von Mitte Mai bis Mitte Juni 2006 erstreckte, wurden Studierende an den ausgewählten Universitäten anhand des entwickelten Fragebogens befragt. Die Interviews wurden von Studierenden, die an zwei Projektlehrveranstaltungen des Instituts für Informationswissenschaft teilnahmen, vorgenommen. ${ }^{38}$ Als Befragungsorte wurden zentrale (Eingangs)bereiche der einzelnen Universitäten ausgewählt, die von allen Studierenden frequentiert werden konnten. ${ }^{39}$

Insgesamt konnten die Planwerte weitgehend erfüllt werden: Am Ende der Erhebungsphase lagen 1.950 ausgefüllte Fragebögen vor, von denen 19 infolge unplausibler Antworten auszuscheiden waren. Anhand von Tabelle 2 lässt sich erkennen, dass alle ausgeschiedenen Fragebögen solche von Studierenden der UG sind. Somit fließen für diese Universität nur $1.081^{40}$ anstelle der 1.100 geplanten Fragebögen in die Resultate ein. ${ }^{41}$

Auf reinem Zufall beruht die Tatsache, dass exakt gleich viele weibliche wie männliche Studierende befragt wurden. Diesbezüglich gab es keiner- lei Vorgaben. Die in Summe ausgewogene Geschlechterverteilung wird auch durch eine universitätsweise Betrachtung relativiert: Der Anteil der weiblichen Studierenden innerhalb der Stichprobe beträgt an der UG 60\%, an der MUG 61\% und an der KUG 52\%. An der TUG liegt der Anteil weiblicher Studierender dagegen nur bei 18\%. ${ }^{42}$ Das Durchschnittsalter der Befragten liegt bei 23 Jahren. Lediglich 3\% der Befragten haben bereits eigene Kinder. Interessanterweise liegt der entsprechende Anteil für die TUG bei $6 \%$, was wiederum am höheren Durchschnittsalter von 24 Jahren der an dieser Universität Studierenden liegen dürfte. Der Anteil der Studienbeihilfenempfänger beträgt 29\%. 37\% der Befragten haben Eltern, die aktuell rauchen. Betrachtet man das Rauchverhalten der einzelnen Elternteile, so zeigt sich, dass in nur 9\% der Fälle beide Elternteile rauchen. in 16\% der Fälle raucht nur der Vater, in 12\% nur die Mutter.

\section{Ergebnisse}

Die Auswertung der erhobenen Daten erfolgte mit Hilfe des Statistikprogrammpaketes „SPSS für Windows“. Die Untersuchungsergebnisse sind in Analogie zu Abschnitt 2 in die Abschnitte „Rauchverhalten“, „Einflussfaktoren" und „Einstellung" untergliedert.

\subsection{Rauchverhalten}

Gemäß Abbildung 1 sind 35\% der Befragten Raucher. Vergleicht man diesen Wert mit jenen für die österreichische Gesamtbevölkerung, so zeigt sich, dass er um 6 Prozentpunkte höher ist als der WHO-Wert aus dem Jahr 2000, aber noch immer um 2 Prozentpunkte niedriger als der Wert in der Studie von Haidinger/Waldhoer/Vutuc (1998), der sich auf das Jahr 1995 bezieht. Demnach scheint sich der Raucheranteil unter den Studierenden nicht wesentlich von jenem innerhalb der Gesamtbevöl-
Tabelle 2: Zusammenfassung der Stichprobe

\begin{tabular}{|lcccc|}
\hline Merkmal & \multicolumn{4}{c|}{ Ausprägung } \\
\hline Universität $(\mathrm{n}=1.931)$ & UG & TUG & MUG & KUG \\
Geschlecht $(\mathrm{n}=1.926)$ & $56 \%(1.081)$ & $23 \%(450)$ & $16 \%(300)$ & $5 \%(100)$ \\
& Weiblich & Männlich & \\
Alter $(\mathrm{n}=1.929)$ & $50 \%(963)$ & $50 \%(963)$ & \\
& Durchschnittsalter: 23 Jahre & \\
Kinder $(\mathrm{n}=1.928)$ & & Nein & \\
& Ja & $97 \%(1.869)$ \\
Studienbeihilfe $(\mathrm{n}=1.927)$ & Ja (59) & Nein \\
Rauchverhalten-Eltern $(\mathrm{n}=1.918)$ & Raucher & Nichtraucher \\
& $37 \%(704)$ & $63 \%(1.214)$ \\
\hline
\end{tabular}

Quelle: eigene Darstellung 
Abbildung 1: Aktuelles Rauchverhalten

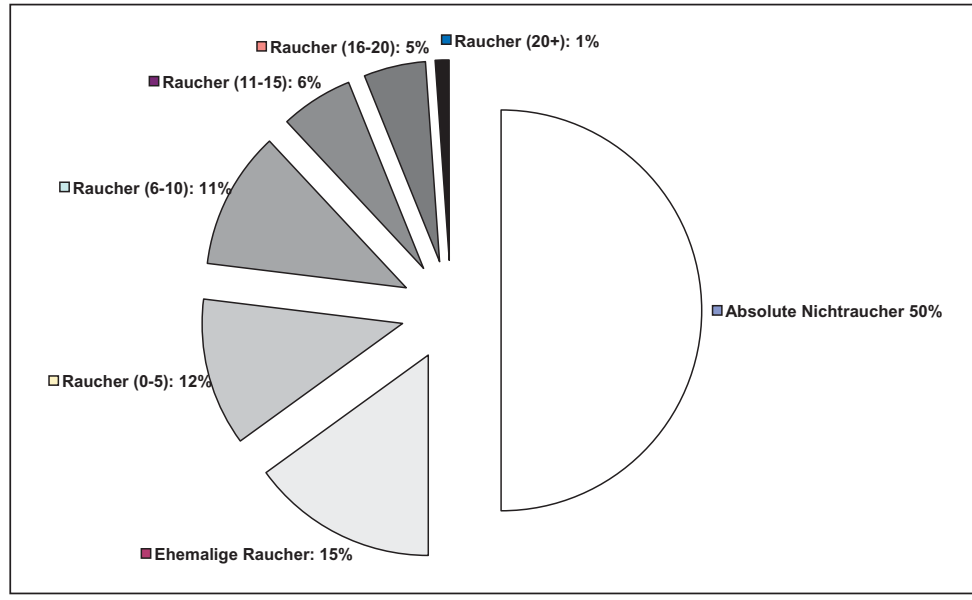

Quelle: eigene Darstellung

kerung zu unterscheiden. Fraglich bleibt dabei, ob die Differenzen zwischen den Untersuchungsergebnissen auf die unterschiedlichen Erhebungszeiträume und/oder die unterschiedlichen Untersuchungsobjekte zurückzuführen sind. Stellt man die Ergebnisse dieser Studie jenen hinsichtlich des Rauchverhaltens von Studierenden in anderen Staaten gegenüber, so ergibt sich erwartungsgemäß ein eher heterogenes Bild. Untersuchungen in den USA haben, was den Raucheranteil unter den Studierenden betrifft, zum Teil zu sehr ähnlichen Ergebnissen geführt. ${ }^{43}$ In anderen Staaten, wie beispielsweise der Tür$\mathrm{kei}^{44}$, ist der Raucheranteil innerhalb der Gruppe der Studierenden dagegen tendenziell höher, während dieser in den skandinavischen Staaten ${ }^{45}$ tendenziell geringer ist.

\section{a. Raucher}

In Abbildung 1 sind die Raucher auf Basis des täglichen Zigarettenkonsums in fünf Klassen unterteilt. Dabei wird ersichtlich, dass die Klassenstärke mit zunehmender Rauchintensität abnimmt. In die Klasse der stärksten Raucher mit einem Zigarettenkonsum von über 20 Zigaretten pro Tag fallen nur mehr 3\% der rauchenden Studierenden. Im Durchschnitt konsumieren die befragten Raucher etwas mehr als 10 Zigaretten pro Tag.

Gemäß Abbildung 2 haben beinahe 5\% der befragten studentischen Raucher bereits vor Vollendung des 14. Lebensjahres, also noch vor Erreichung der Mündigkeit, mit dem Rauchen begonnen. Diesbezüglich sollten jedenfalls verstärkt Maßnahmen ergriffen werden, die sich nicht nur auf Aufklärungskampagnen beschränken dürften, sondern in Form gesetzlicher Verbote und vor allem Sanktionen erfolgen müssten. Die Sanktionen sollten sich dabei in erster Linie gegen (erwachsene) Personen richten, die den unmündigen Minderjährigen den Erwerb bzw. Konsum von Tabakwaren in direkter ${ }^{46}$ oder indirekter ${ }^{47}$ Form ermöglichen. Für Jugendliche zwischen 14 und 17 Jahren würden sich hingegen verstärkt Aufklärungsmaßnahmen anbieten, da man in diesem Alter bereits eine erhöhte Einsichtsfähigkeit und Eigenverantwortlichkeit erwarten kann. Flankierende gesetzliche Maßnahmen erscheinen aber auch für diese Altersgruppe unabdingbar. Allerdings dürften die bestehenden Jugendschutzmaßnahmen im Hinblick auf das Rauchen nicht immer die erwünschte Wirkung zeigen, da immerhin $26 \%$ der Befragten vor Vollendung des 16 . Lebensjahres zu rauchen begonnen haben. Eventuell ist die mittlerweile eingeführte Alterskontrolle für den Erwerb von Tabakwaren an Automaten ein Schritt in die richtige Richtung. Die meisten Studierenden haben im Alter von 16 bzw. 17 Jahren mit dem Rauchen begonnen. Dies spricht für eine grundsätzliche Wirksamkeit der Jugendschutzmaßnahmen, die für Jugendliche bis zur Vollendung des 16 . Lebensjahres vorgesehen sind. Deshalb wäre es durchaus überlegenswert, die Geltung bestehender und möglicher zukünftiger Jugendschutzmaßnahmen auf Jugendliche bis zur Erreichung der Volljährigkeit auszudehnen. Eine derartige Regelung existiert beispielsweise in Schweden ${ }^{48} .{ }^{49}$ Argumentativ schwierig erscheint eine solche Ausdehnung in Österreich vor dem Hintergrund der bereits erfolgten Senkung des Wahlalters auf 16 Jahre: Kann man Jugendlichen, denen man bereits politische Entscheidungsfähigkeit zutraut, die Entscheidungsfähigkeit im Hinblick auf den Erwerb und Konsum von Tabakwaren absprechen?

Insgesamt hat somit die Mehrzahl der studentischen Raucher bereits vor Aufnahme des Studiums mit dem

Abbildung 2: Rauchbeginn ${ }^{50}$

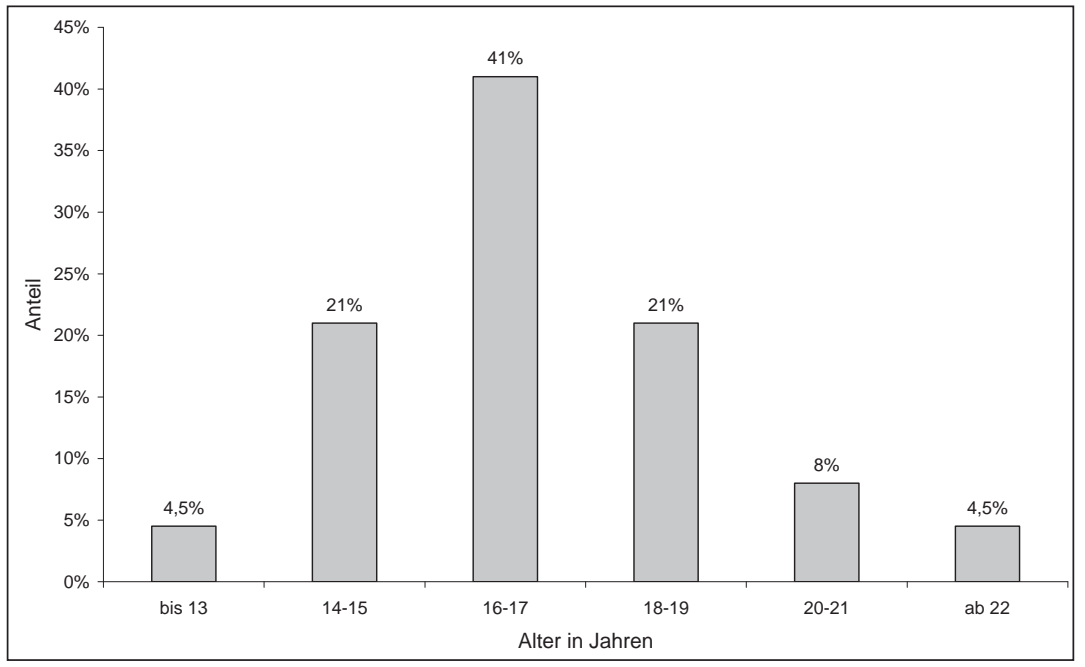

Erläuterung zu den Altersklassen: „bis 13” bedeutet von der Geburt bis einen Tag vor dem 14. Geburtstag, "14-15" bedeutet vom 14. Geburtstag bis einen Tag vor dem 16. Geburtstag, usw. Quelle: eigene Darstellung 
Rauchen begonnen. Wie viele Personen in der Altersklasse „18-19“ anlässlich der Erreichung ihrer Volljährigkeit, wie viele anlässlich ihres Studienbeginns ${ }^{51}$ und wie viele aus anderen Anlässen mit dem Rauchen angefangen haben, lässt sich im Rahmen der vorliegenden Untersuchung nicht klären. Untersuchungen in den USA haben gezeigt, dass die neue universitäre Umgebung oftmals einen deutlich negativen Einfluss auf das Rauchverhalten angehender Akademiker ausübt. ${ }^{52}$ Nichtraucherkampagnen für Studienanfänger können somit keinesfalls schaden. Die Zahlen hinsichtlich der beiden höchsten Altersklassen in Abbildung 2 deuten darauf hin, dass die Wahrscheinlichkeit, mit dem Rauchen zu beginnen, mit zunehmendem Alter deutlich abnimmt. Dies ist jedoch vor dem Hintergrund der Zusammensetzung der Stichprobe zu interpretieren, da beinahe die Hälfte der Befragten zum Zeitpunkt der Befragung das 22. Lebensjahr noch nicht vollendet hatte.

Aus Tabelle 3 geht hervor, dass beinahe die Hälfte der befragten Raucher mit dem Rauchen aufhören möchte. Für diese Gruppe bieten sich insbesondere Unterstützungsmaßnahmen, wie etwa eine ambulante oder sogar stationäre Rauchertherapie, an. Angehörige der Gruppe der „überzeugten“ Raucher könnten eventuell durch eine intensive Konfrontation mit den negativen gesundheitlichen Folgen des Rauchens zu einem Umdenken bewogen werden. Eine solche Konfrontation könnte in Form von Radio- oder Fernsehspots erfolgen, in denen infolge des Tabakkonsums erkrankte Raucher über ihren Gesundheitszustand berichten. ${ }^{53}$ Als weitere Maßnahme zur Veränderung des aktuellen Rauchverhaltens beider Gruppen käme die Einführung von höheren Beiträgen zur sozialen (und auch privaten) Krankenversicherung für Raucher in Frage. ${ }^{54}$ Laut Tabelle 3 ist der Wunsch von Rauchern, mit dem Rauchen aufzuhören, kaum davon abhängig, seit wie vielen Jahren sie bereits rauchen. Das Ausmaß des täglichen Zigarettenkonsums spielt für diesen Wunsch dagegen sehr wohl eine Rolle ${ }^{55}$ : Unter Studierenden, die sehr wenig rauchen, ist der Wunsch etwas schwächer, unter jenen, die sehr viel rauchen, deutlich schwächer ausgeprägt. Der Grund dafür könnte im ersten Fall in der von den Betroffenen als gering eingeschätzten Gesundheitsgefährdung, im zweiten Fall in einer ausgeprägten Nikotinabhängigkeit zu finden sein.

Jeweils mehr als zwei Drittel der befragten Raucher gaben an, aus Gewohnheit bzw. zur Entspannung und Stressbewältigung zu rauchen (vgl. Abbildung 3). Dies deutet auf eine gewisse Nikotinabhängigkeit der Raucher hin, was wiederum ein verstärktes Angebot an Entwöhnungsmaßnahmen auf Kosten der Sozialversicherung rechtfertigen würde. Erfolgt das Rauchen dagegen in erster Linie zum Zeitvertreib, könnten Preiserhöhungen für Tabakwaren ein probates Mittel sein, um derart motivierte Raucher zu einem gesünderen Zeitvertreib zu bewegen. Erstaunlicherweise spielt der Geschmack als Grund für das Rauchen eine relativ große Rolle. Personen, die aus diesem Grund rauchen, empfinden Rauch-
Tabelle 3: Wille zum Aufhören

\begin{tabular}{|lllcc|}
\hline Einflussfaktor & Ausprägung & \multicolumn{3}{c|}{ Anteil der Befragten } \\
& & Wille zum Aufhören & $\begin{array}{c}\text { Korrelations- } \\
\text { koeffizient }\end{array}$ \\
\hline Ja & Nein & $55 \%$ & \\
Zigarettenkonsum & Gesamt & $41 \%$ & $59 \%$ & \\
pro Tag & bis Stück & $49 \%$ & $51 \%$ & 0,03 \\
& 6-10 Stück & $47 \%$ & $53 \%$ & \\
& 11-15 Stück & $47 \%$ & $53 \%$ & \\
& 16-20 Stück & $28 \%$ & $72 \%$ & \\
über 20 Stück & $42 \%$ & $58 \%$ & 0,03 \\
Dauer des aktuellen & bis 2 Jahre & $48 \%$ & $52 \%$ & \\
Rauchverhaltens & 3-5 Jahre & $43 \%$ & $57 \%$ & \\
& 6-10 Jahre & $43 \%$ & $57 \%$ & \\
& über 10 Jahre & & & \\
\hline
\end{tabular}

Quelle: eigene Darstellung

verbote vermutlich als starken Eingriff in ihre persönliche Lebensqualität und dürften diesen dementsprechend skeptisch gegenüberstehen. Nur 7\% der Befragten gaben an, infolge Gruppenzwangs zu rauchen. Für eine noch kleinere Anzahl stellt das Rauchen ein Mittel zur „Regulierung“ des Körpergewichts dar. ${ }^{56}$ In den USA spielt dieser Grund hingegen eine wesentlich größere Rolle. ${ }^{57}$ Insgesamt ist hinsichtlich der Ergebnisse zu den Gründen für das Rauchen zu beachten, dass Mehrfachnennungen möglich waren. Somit lässt sich im Rahmen der gegenständlichen Untersuchung nicht sagen, welcher Grund für das Rauchen jeweils der wichtigste ist. ${ }^{58}$ Die Erstellung einer diesbezüglichen Rangliste könnte Inhalt einer Folgeuntersuchung sein.

\section{b. Nichtraucher}

Innerhalb der Gruppe der nichtrauchenden Studierenden bilden jene Personen, die nie geraucht haben, mit 76\% eindeutig die Mehrheit (vgl. Abbildung 1). Insgesamt ${ }^{59}$ beträgt der Anteil dieser „,absoluten “ Nichtraucher 50\%. Dieses Ergebnis unterscheidet sich damit kaum vom entsprechenden Wert für die österreichische Gesamtbevölkerung, der 1995 bei rund 51\% lag. ${ }^{60}$

Für das aktuelle Rauchverhalten von studentischen Nichtrauchern spielen eindeutig gesundheitliche Aspekte die größte Rolle (vgl. Tabelle 4). 82\% der befragten Nichtraucher gaben an, aus gesundheitlichen Überlegungen nicht zu rauchen. Unterteilt man diese Gruppe in „absolute" Nichtraucher und ehemalige Raucher, so lässt der wesentlich höhere Prozentwert bei den ehemaligen Rauchern darauf schließen, dass vorwiegend gesundheitliche Aspekte für deren Wandlung zu Nichtrauchern ausschlaggebend gewesen sein dürften. Kostenüberlegungen stellen insgesamt immerhin den zweiten wichtigen Grund für das Nichtrauchen dar. Diese Resultate deuten darauf hin, dass sich Präventionsmaßnahmen und Maßnahmen zur Änderung des Rauchverhaltens von Rauchern schwerpunktmäßig auf Gesundheits- und Kos- 
Abbildung 3:

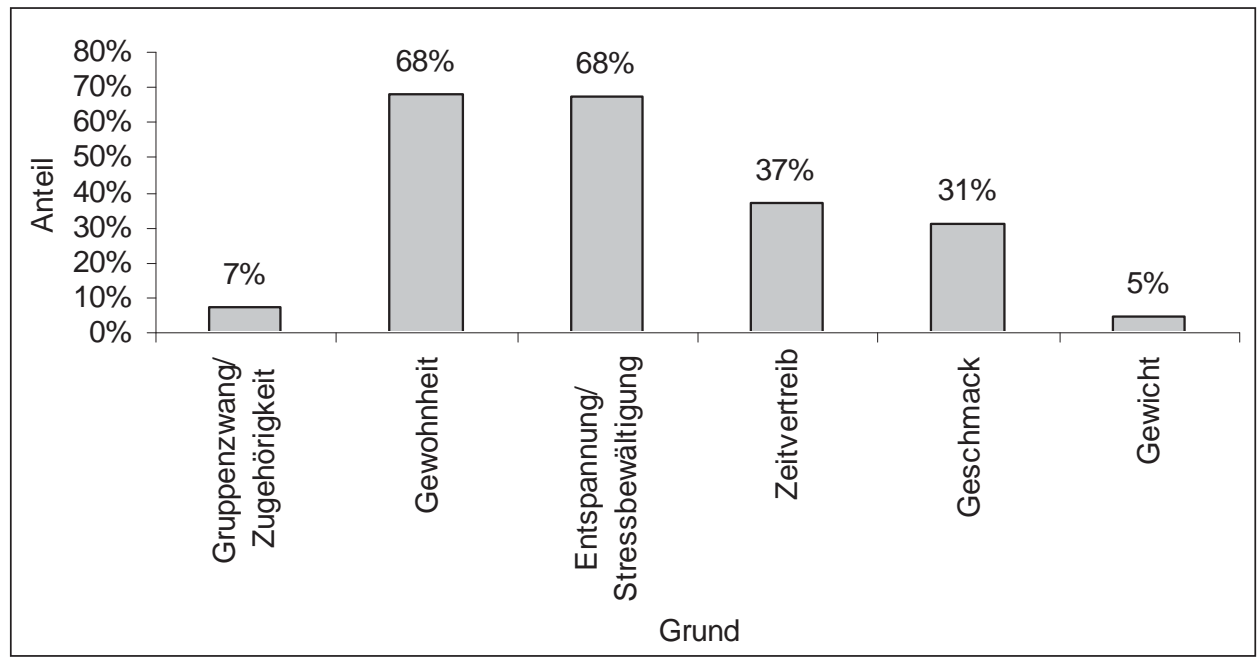

Quelle: eigene Darstellung

tenaspekte beziehen sollten. ${ }^{61}$ Dagegen scheinen gesetzliche Rauchverbote und Warnhinweise auf Zigarettenpackungen für das aktuelle Rauchverhalten von Nichtrauchern nur von untergeordneter Bedeutung zu sein. ${ }^{62}$ Dasselbe gilt für den gesellschaftlichen Trend zum Nichtrauchen.

\subsection{Einflussfaktoren}

Anhand von Tabelle 5 lässt sich erkennen, dass die untersuchten soziodemographischen Faktoren bzw. Rahmenbedingungen sehr wohl einen gewissen Einfluss auf das Rauchverhalten der befragten Studierenden ausüben. Bezüglich des Faktors „Universität“ wird die eingangs geäußerte Vermutung bestätigt, dass Studierende an MU weniger häufig rauchen, da es an der MUG überdurchschnittlich viele Nichtraucher gibt. Betrachtet man die übrigen drei untersuchten Universitäten, so stehen den $72 \%$ Nichtrauchern an der MUG 64\% an der $\mathrm{UG}^{63}, 65 \%$ an der TUG und $61 \%$ an der KUG gegenüber. Ebenfalls den Erwartungen entspricht der jeweils höhere Nichtraucheranteil unter den weiblichen Studierenden, den jün-

Tabelle 4: Gründe für das Nichtrauchen

\begin{tabular}{|c|c|c|c|c|}
\hline \multirow[t]{2}{*}{ Grund fürs Nichtrauchen } & \multicolumn{3}{|c|}{ Nennungen } & \multirow[b]{2}{*}{$\begin{array}{r}\text { Kontingenz- } \\
\text { koeffizient }\end{array}$} \\
\hline & $\begin{array}{c}\text { Nichtraucher } \\
\text { Gesamt }\end{array}$ & $\begin{array}{c}\text { Absolute } \\
\text { Nichtraucher }\end{array}$ & $\begin{array}{c}\text { Ehemalige } \\
\text { Raucher }\end{array}$ & \\
\hline Gesundheit & $82 \%$ & $81 \%$ & $89 \%$ & 0,08 \\
\hline Kosten & $58 \%$ & $57 \%$ & $62 \%$ & 0,05 \\
\hline Gesetzliche Rauchverbote & $2 \%$ & $2 \%$ & $2 \%$ & 0,01 \\
\hline Warnhinweise & $3 \%$ & $4 \%$ & $0,3 \%$ & 0,09 \\
\hline Gesellschaftlicher Trend & $3 \%$ & $4 \%$ & $3 \%$ & 0,02 \\
\hline
\end{tabular}

Quelle: eigene Darstellung geren ${ }^{64}$ Studierenden, den Studierenden mit Kind sowie den Studienbeihilfenbeziehern.

Der bedeutendste der betrachteten Einflussfaktoren ist zweifellos das Rauchverhalten der Eltern. Studierende, deren Eltern Nichtraucher sind, sind zu 71\% Nichtraucher; Studierende, bei denen zumindest ein Elternteil raucht, nur zu 55\% ${ }^{65}$. Die Relevanz dieses Einflussfaktors wurde - wie bereits erwähnt - schon in anderen Untersuchungen festgestellt und stellt somit keine große Überraschung dar. Infolge dieser wichtigen positiven oder auch negativen Vorbildwirkung der Eltern sollten Maßnahmen zur Eindämmung des Rauchens unter Jugendlichen durchaus auf deren Eltern gerichtet sein. In diesem Zusammenhang stellt sich die Frage, ob ein Rauchverbot für Privatwohnungen, in denen Kinder leben, nicht eine sinnvolle derartige Maßnahme darstellen würde.

\subsection{Einstellung}

Wie aus Tabelle 6 hervorgeht, schätzen Studierende die Schädlichkeit des Aktiv- sowie des Passivrauchens beinahe einhellig richtig ein. Der Informationsstand hinsichtlich der Schädlichkeit des Rauchens dürfte also ausreichend sein. 98\% der Befragten stimmen der Aussage „Ich halte Rauchen für gesundheitsschädlich!“ (Aussage 1) zu, 95\% der Aussage „Ich halte Passivrauchen für gesundheitsschädlich!“ (Aussage 2). Nur 1\% bzw. 2\% der Befragten lehnt bzw. lehnen die Aussagen jeweils ab, der Rest ist neutral. Unterteilt man die Ergebnisse nach dem aktuellen Rauchverhalten der Befragten, so ergeben sich hinsichtlich der zentralen Tendenz keine Unterschiede zwischen Rauchern und Nichtrauchern. Der Median liegt für beide Gruppen hinsichtlich beider Aussagen bei starker Zustimmung. Erst eine Betrachtung der Detailergebnisse offenbart doch gewisse Unterschiede: Während Nichtraucher der Aussage $1 \mathrm{zu}$ 94\% und der Aussage 2 zu 87\% stark zustimmen, liegen die entsprechende Werte für die Gruppe der Raucher bei nur $85 \%$ bzw. $71 \%$.

Der Aussage „Raucher sollten zum Nichtrauchen ermuntert werden!“ 
Tabelle 5: Einflussfaktoren

\begin{tabular}{|c|c|c|c|c|}
\hline \multirow[t]{2}{*}{ Einflussfaktor } & \multirow[t]{2}{*}{ Ausprägung } & \multicolumn{3}{|c|}{ Anteil der Befragten } \\
\hline & & Raucher & Nichtraucher & $\begin{array}{c}\text { Kontingenz- } \\
\text { koeffizient }\end{array}$ \\
\hline \multirow[t]{2}{*}{ Universität } & MU & $28 \%$ & $72 \%$ & \multirow{2}{*}{0,06} \\
\hline & Sonstige Unis & $36 \%$ & $64 \%$ & \\
\hline \multirow[t]{2}{*}{ Geschlecht } & Weiblich & $33 \%$ & $68 \%$ & \multirow{2}{*}{0,05} \\
\hline & Männlich & $37 \%$ & $63 \%$ & \\
\hline \multirow[t]{2}{*}{ Alter } & Jünger & $33 \%$ & $67 \%$ & \multirow{2}{*}{0,03} \\
\hline & Älter & $36 \%$ & $64 \%$ & \\
\hline \multirow[t]{2}{*}{ Kinder } & $\mathrm{Ja}$ & $31 \%$ & $70 \%$ & \multirow{2}{*}{0,02} \\
\hline & Nein & $35 \%$ & $65 \%$ & \\
\hline \multirow[t]{2}{*}{ Studienbeihilfe } & $\mathrm{Ja}$ & $32 \%$ & $68 \%$ & \multirow{2}{*}{0,03} \\
\hline & Nein & $36 \%$ & $64 \%$ & \\
\hline \multirow[t]{2}{*}{ Rauchverhalten-Eltern } & Raucher & $45 \%$ & $55 \%$ & \multirow{2}{*}{0,16} \\
\hline & Nichtraucher & $29 \%$ & $71 \%$ & \\
\hline
\end{tabular}

Quelle: eigene Darstellung

(Aussage 3) stimmen insgesamt 64\% der Befragten zu. Erwartungsgemäß groß sind hier die Unterschiede zwischen Rauchern und Nichtrauchern: 70\% der Nichtraucher, aber nur 50\% der Raucher stehen dieser Aussage positiv gegenüber. Der Median liegt für Nichtraucher sogar bei starker Zustimmung, für Raucher bei Neutralität und insgesamt bei geringer Zustimmung. man vermutlich der Meinung dieser Gruppe größeren Wert beimessen. Überraschend gering sind dagegen die Unterschiede in der Beurteilung der Intoleranz von Nichtrauchern (Aussage 5). Aussage 5 stehen 20\% der Raucher und $17 \%$ der Nichtraucher positiv sowie 48\% der Raucher und 51\% der Nichtraucher negativ gegenüber. Möglicherweise würde eine Zunahme von Rauchverboten, verbunden mit einer rigorosen Kontrolle dieser Verbote, auch hier zu größeren Differenzen führen.

\section{Resümee}

Ziel der gegenständlichen Untersuchung war es, einen ersten detaillierteren Einblick in das Rauchverhalten von Studierenden in Österreich zu geben. Dies sollte infolge der entsprechenden inhaltlichen Gestaltung des eingesetzten Fragebogens sowie des großen Umfanges der Stichprobe gelungen sein. Die Untersuchung legt offen, dass von den 1.931 befragten Studierenden 65\% Nichtraucher sind. 50\% der Befragten haben nie geraucht, 15\% haben damit aufgehört. Würden zusätzlich alle Raucher, die angeben, mit dem Rauchen aufhören zu wollen, ihren Vorsatz verwirklichen, würde der Prozentsatz der Nichtraucher auf $81 \%$ steigen. Somit bilden die „überzeugten“ Raucher aber immerhin noch eine Gruppe
Am größten sind die Differenzen zwischen Rauchern und Nichtrauchern hinsichtlich der Einschätzung der Rücksichtslosigkeit von Rauchern (Aussage 4). Während nur 14\% der Raucher eine gewisse Rücksichtslosigkeit von Rauchern - und damit bis zu einem bestimmten Grad auch von sich selbst - gegenüber Nichtrauchern bestätigen, aber 58\% diese Behauptung zurückweisen, liegen die entsprechenden Werte für die Gruppe der Nichtraucher bei 37\% bzw. 29\%. Wenn es um die Einführung von Maßnahmen zum Schutz von Nichtrauchern geht, wird
Tabelle 6: Einstellung

\begin{tabular}{|c|c|c|c|c|c|c|c|c|}
\hline \multirow{2}{*}{ Gegenstand } & \multirow{2}{*}{ Gruppe } & \multicolumn{5}{|c|}{ Grad der Zustimmung } & \multirow{2}{*}{ Median } & \multirow{2}{*}{$\begin{array}{c}\text { Korrelations- } \\
\text { koeffizient }\end{array}$} \\
\hline & & ++ & + & $\mathrm{n}$ & - & -- & & \\
\hline \multirow{3}{*}{$\begin{array}{l}\text { Schädlichkeit des } \\
\text { Rauchens }\end{array}$} & Gesamt & $91 \%$ & $7 \%$ & $2 \%$ & $1 \%$ & $0 \%$ & ++ & \\
\hline & Raucher & $85 \%$ & $12 \%$ & $3 \%$ & $1 \%$ & $0 \%$ & ++ & 0,161 \\
\hline & Nichtraucher & $94 \%$ & $4 \%$ & $1 \%$ & $0 \%$ & $0 \%$ & ++ & \\
\hline \multirow{3}{*}{$\begin{array}{l}\text { Schädlichkeit des } \\
\text { Passivrauchens }\end{array}$} & Gesamt & $81 \%$ & $14 \%$ & $4 \%$ & $1 \%$ & $1 \%$ & ++ & \\
\hline & Raucher & $71 \%$ & $19 \%$ & $7 \%$ & $1 \%$ & $1 \%$ & ++ & 0,194 \\
\hline & Nichtraucher & $87 \%$ & $11 \%$ & $2 \%$ & $0 \%$ & $1 \%$ & ++ & \\
\hline \multirow{3}{*}{$\begin{array}{l}\text { Ermunterung zum } \\
\text { Nichtrauchen }\end{array}$} & Gesamt & $43 \%$ & $21 \%$ & $25 \%$ & $4 \%$ & $8 \%$ & + & \\
\hline & Raucher & $29 \%$ & $21 \%$ & $31 \%$ & $7 \%$ & $13 \%$ & $\mathrm{n}$ & 0,226 \\
\hline & Nichtraucher & $50 \%$ & $20 \%$ & $22 \%$ & $3 \%$ & $5 \%$ & ++ & \\
\hline \multirow{3}{*}{$\begin{array}{l}\text { Rücksichtslosigkeit } \\
\text { von Rauchern }\end{array}$} & Gesamt & $13 \%$ & $17 \%$ & $32 \%$ & $17 \%$ & $22 \%$ & $\mathrm{n}$ & \\
\hline & Raucher & $4 \%$ & $10 \%$ & $27 \%$ & $22 \%$ & $36 \%$ & - & 0,315 \\
\hline & Nichtraucher & $17 \%$ & $20 \%$ & $35 \%$ & $14 \%$ & $15 \%$ & $\mathrm{n}$ & \\
\hline \multirow{3}{*}{$\begin{array}{l}\text { Intoleranz von } \\
\text { Nichtrauchern }\end{array}$} & Gesamt & $5 \%$ & $13 \%$ & $32 \%$ & $18 \%$ & $32 \%$ & $\mathrm{n}$ & \\
\hline & Raucher & $6 \%$ & $14 \%$ & $32 \%$ & $20 \%$ & $28 \%$ & $\mathrm{n}$ & 0,054 \\
\hline & Nichtraucher & $4 \%$ & $13 \%$ & $32 \%$ & $16 \%$ & $35 \%$ & - & \\
\hline
\end{tabular}

Quelle: eigene Darstellung 
von 19\%. 41\% der Raucher haben nach Vollendung des 16. Lebensjahres, aber vor Erreichung der Volljährigkeit zu rauchen begonnen. Präventionsmaßnahmen sollten daher verstärkt auf diese Altersgruppe gerichtet werden. Ebenso sollten sich Maßnahmen zur Eindämmung des Rauchens unter jungen Erwachsenen verstärkt an rauchende Eltern richten, da deren Rauchverhalten einen deutlich negativen Einfluss auf das Rauchverhalten ihrer Kinder ausübt. Die Untersuchung der Gründe für das aktuelle Rauchverhalten hat die hohe Bedeutung gesundheitlicher sowie finanzieller Aspekte offen gelegt. Hier könnten weitere Maßnahmen zur Eindämmung des Rauchens ansetzen.

\section{Literatur}

Ahmad, Sajjad (2005), Closing the youth access gap: The projected health benefits and cost savings of a national policy to raise the legal smoking age to 21 in the United States, in: Health Policy, 75. Jg., Nr. 1, S. 74-84.

Biasco, Frank und James P. Hartnett (2002), Colleges Students' Attitudes Toward Smoking, in: College Student Journal, 36. Jg., Nr. 3, S. 442 447.

Bricker, Jonathan, Brian Leroux, M. Robyn Andersen, Kumar Bharat Rajan, und Arthur Peterson Jr. (2005), Parental smoking cessation and children's smoking: Mediation by antismoking actions, in: Nicotine \& Tobacco Research, 7. Jg., Nr. 4, S. 501-509.

Cawley, John, Sarah Markowitz und John Tauras (2004), Lighting up and slimming down: the effects of body weight and cigarette prices on adolescent smoking initiation, in: Journal of Health Economics, 23. Jg., Nr. 2, S. 293-311.

Chaaya, Monique, Zana El Roueiheb, Hiam Chemaitelly, Grace Azar, Joumana Nasr und Ban Al-Sahab (2004), Argileh smoking among university students: A new tobacco epidemic, in: Nicotine \& Tobacco Research, 6. Jg., Nr. Heft 3, S. 457-463.

Chalmers, Karen, Marilyn Seguire und Judy Brown (2002), Tobacco use and baccalaureate nursing students: a study of their attitudes, beliefs and personal behaviours, in: Journal of Advanced Nursing, 40. Jg., Nr. 1, S. 17-24.

Emmons, Karen, Henry Wechsler, George Dowdall und Melissa Abraham (1998), Predictors of smoking among US college students, in American Journal of Public Health, 88. Jg., Nr. 1, S. 104-107.

Gilpin, Elizabeth, Martha White, Arthur Farkas und John Pierce (1999), Home smoking restrictions: which smokers have them and how they are associated with smoking behavior, in: Nicotine \& Tobacco Research, 1. Jg., Nr. 2, S. 153-162.

Haidinger, Gerald, Thomas Waldhoer und Christian Vutuc (1998), The Prevalence of Smoking in Austria, in: Preventive Medicine, 27. Jg., Nr. 1, S. 50-55.

Hammar, Henrik und Fredrik Carlsson (2005), Smokers‘ Expectations to Quit Smoking, in: Health Economics, 14. Jg., Nr. 3, S. 257-67.

Hammond, David, Geoffrey Fong, Ron Borland, Michael Cummings, Ann McNeill und Pete Driezen (2007), Text and Graphic Warnings on Cigarette Packages. Findings from the International Tobacco Control Four Country Study, in: American Journal of Preventive Medicine, 32. Jg., Nr. 3, S. 202-209.

Kürsten, Reinhard (2006), Passivrauchen - Tödliche Gefahr, in: Österreichische Ärztezeitung vom 25. Mai 2006, S. 60-69.

Kunze, Michael, Ernest Groman und Ursula Kunze (2004), Tobacco consumption and tobacco-related diseases: gender differences with a comparison between two European countries, in: The Journal of Men's Health \& Gender, 1. Jg., Nr. 1, S. 83-87.

Loukas, Alexandra, Marcie Garcia und Nell Gottlieb (2006), Texas College Students' Opinions of No-Smoking Policies, Secondhand Smoke, and Smoking in Public Places, in: Journal of American College Health, 55. Jg., Nr. 1, S. 27-32.
Metinta, Selma, Mehmet Sariboyac, S. Nuho lu, Muzaffer Metinta , Cemal Kalyoncu, S. Etiz, Nihan Özdemir und Can Akta (1998), Smoking patterns of university students in Eski ehir, Turkey, in: Public Health (Nature), 112. Jg., Nr. 4, S. 261-264.

Nicolas, Angel (2002), How Important Are Tobacco Prices in the Propensity to Start and Quit Smoking? An Analysis of Smoking Histories from the Spanish National Health Survey, in: Health Economics, 11. Jg., Nr. 6, S. 521-35.

Okuyemi, Kolawole, Jasjit Ahluwalia, Kimber Richter, Mathew Mayo und Ken Resnicow (2001), Differences among African American light, moderate, and heavy smokers, in: Nicotine \& Tobacco Research, 3. Jg., Nr. 1, S. 45-50.

Pechmann, Cornelia und Ellen Reibling (2006), Antismoking Advertisements for Youths: An Independent Evaluation of Health, Counter-Industry, and Industry Approaches, in: American Journal of Public Health, 96. Jg., Nr. 5, S. 906-913.

Pentz, Mary (1999), Effective prevention programs for tobacco use, in: Nicotine \& Tobacco Research, 1. Jg. Supplement 2, S. S99-S107.

Ridner, S. Lee (2005), Predicting Smoking Status in a College-Age Population, in: Public Health Nursing, 22. Jg., Nr. 6, S. 494-505.

Saffer, Henry und Frank Chaloupka (2000), The effect of tobacco advertising bans on tobacco consumption, in: Journal of Health Economics, 19. Jg., Nr. 6, S. 1117-1137.

Samuels, Noah (1997), Smoking among hospital doctors in Israel and their attitudes regarding anti-smoking legislation, in: Public Health, 111. Jg., Nr. 5, S. 285-288

Sopp, Alexander (2005), Tabakkonsum: Kosten, Nutzen, Werbeverbote, in: Gesundheits- und Sozialpolitik, Nr. 9-10, S. 42-50.

Sundh, Mona und Curt Hagquist (2005), Effects of a minimum-age tobacco law - Swedish experience, in: Drugs: Education, Prevention \& Policy, 12. Jg., Nr. 6, S. 501-510.

Waters, Kimberly, Kari Harris, Sandra Hall, Niaman Nazir und Alex Waigandt (2006), Characteristics of Social Smoking Among College Students, in: Journal of American College Health, 55. Jg., Nr. 3, S. 133-139.

Wegner, Christoph, Andreas Gutsch, Franz Hessel und Jürgen Wasem (2005), Die Kosten der tabakbedingten Inanspruchnahme von Gesundheitsleistungen in Deutschland, in: Gesundheits- und Sozialpolitik, Nr. 9-10, S. 34-41.

\section{Fußnoten}

1 An dieser Studie haben folgende Studierende der Karl-FranzensUniversität Graz mitgewirkt: Herr Fouad Baradaran-Lilabadi, Herr Clemens Juriga-Lamut, Herr Günther Kainz, Frau Katrin Kall, Frau Sandra Kernmaier, Frau Claudia Kropiunig, Frau Aldina Kumalic, Frau Elisabeth Pachler, Herr Christian Radauer, Frau Elisabeth Schützenhöfer sowie Herr Reinhard Welser.

2 Vgl. Kürsten (2006).

3 Vgl. Forum Prävention, Seite I, in: Soziale Sicherheit 1/2002.

4 Zu Präventionsmaßnahmen im Allgemeinen vgl. Pentz (1999).

5 Vgl. §§5f. Tabakgesetz (Bundesgesetz über das Herstellen und das Inverkehrbringen von Tabakerzeugnissen sowie die Werbung für Tabakerzeugnisse und den Nichtraucherschutz, BGBl. Nr. 431/1995, zuletzt geändert durch BGBl. I Nr. 47/2006).

6 Vgl. §11 Tabakgesetz. Zur Wirkung derartiger Werbe- und Sponsoringverbote vgl. z.B. Saffer/Chaloupka (2000).

7 Vgl. z.B. \$9 Steiermärkisches Jugendschutzgesetz (Gesetz vom 7. Juli 1998 über den Schutz der Jugend - StJSchG): Demnach sind der Konsum und die Abgabe von Tabakwaren an Personen bis zum vollendeten 16. Lebensjahr verboten.

8 Vgl. Kürsten (2006).

9 Vgl. §30 AschG (Bundesgesetz über Sicherheit und Gesundheitsschutz bei der Arbeit - ArbeitnehmerInnenschutzgesetz, BGBl. Nr. 497/1995, zuletzt geändert durch BGBl. II Nr. 13/2007).

10 Vgl. §13 Tabakgesetz.

11 Vgl. Vereinbarung zur Einrichtung von Nichtraucherbereichen in Gastgewerbebetrieben, abgeschlossen zwischen dem Gesundheitsministerium und dem Fachverband Gastronomie im Jahre 2004. Demnach verzichtet das Bundesministerium auf eine gesetzliche 
Regelung des Nichtraucherschutzes im Gastgewerbe, sofern auf freiwilliger Basis „Speisebetriebe“ ab einer Fläche (Gästebereich) von $75 \mathrm{~m}^{2}$ einen Nichtraucherbereich einrichten, der mindestens $40 \%$ der Sitzplätze umfasst.

12 Vgl. z.B. den Beitrag „Eine Form der Kindesmisshandlung“ in der österreichischen Tageszeitung „Der Standard“ vom 4.12.2006: In diesem wird Rauchen in Gegenwart von kleinen Kindern plakativ als „eine Form der Kindesmisshandlung“ bezeichnet.

13 Vgl. Haidinger/Waldhoer/Vutuc (1998) und Kunze/Groman/Kunze (2004).

$14 \mathrm{Vgl}$. WHO (26.1.2007), European health for all database (HFA-DB), http.//data.euro.who.int/hfadb/.

15 Im ebenfalls von der WHO herausgegebenen "European Tobacco Control Report 2007" sind zwar neuere Daten zum Rauchverhalten enthalten, doch handelt es sich dabei zu einem erheblichen Teil nur um Schätzwerte.

16 Stand: 2000.

17 Stand: 2000.

18 Die entsprechenden Werte für Deutschland für das Jahr 2000 liegen bei 35\% (für Deutschland gibt es auch einen diesbezüglichen Wert für das Jahr 2003, der bei 34\% liegt) bzw. 1.553 Zigaretten.

19 Vgl. etwa Chaaya u.a. (2004) für den Libanon oder auch Metinta u.a. (1998) für die Türkei.

20 Studierende der Montanistischen Universität Leoben, welche zum Typus Technische Universität gezählt wird, wurden nicht befragt.

21 Vgl. Metinta u.a. (1998).

22 So etwa durch Aktivitäten der Sozialversicherung im Bereich der stationären und ambulanten Raucherentwöhnung (vgl. Forum Prävention, Seiten XXV-XXVII, in: Soziale Sicherheit 9/2003).

23 Vgl. Hammar/Carlsson (2005).

24 Vgl. z.B. Emmons u.a. (1998).

25 Vgl. Metinta u.a. (1998) und Ridner (2005).

26 Vgl. Samuels (1997).

27 Vgl. Haidinger/Waldhoer/Vutuc (1998).

28 Vgl. Gilpin u.a. (1999)

29 Vgl. Bricker u.a. (2005) und Ridner (2005).

30 Vg. z.B. Kürsten (2006).

31 In die Gestaltung des endgültigen Fragebogens flossen auch die Ergebnisse eines Pretests ein, der Anfang Mai 2006 an der KarlFranzens-Universität Graz durchgeführt wurde.

32 Vgl. Chaaya u.a. (2004) und Waters u.a. (2006)

33 Vgl. z.B. Chalmers/Seguire/Brown (2002).

34 Im Fragebogen sind als Beispiele das Rauchverbot am Arbeitsplatz im Falle von nichtrauchenden Kollegen im gleichen Raum sowie das Rauchverbot in öffentlichen Gebäuden genannt.

35 Diesbezüglich werden im Fragebogen Warnhinweise in der Werbung oder auf den Zigarettenpackungen als Beispiele angeführt.

36 Im Fragebogen wird dieser Trend als Tendenz zur Stigmatisierung der Raucher konkretisiert.

37 Und zwar 1.100 Studierende der UG (insgesamt ca. 22.000 Studierende), 450 der TUG (insgesamt ca. 9.000 Studierende), 300 der MUG (insgesamt ca. 6.000 Studierende) sowie 100 der KUG (insgesamt ca. 2.000 Studierende). Von den 1.100 Studierenden der UG sollten entsprechend der Verteilung der Studierenden auf die einzelnen Fakultäten 375 der Geisteswissenschaftlichen (GEWI), jeweils 250 der Naturwissenschaftlichen (NAWI) sowie der Sozial- und Wirtschaftswissenschaftlichen (SOWI), 200 der Rechtswissenschaftlichen (REWI) und 25 der Theologischen (THEO) Fakultät befragt werden.

38 Am Projekt „Studierende und Rauchen“ waren elf Studierende beteiligt (vgl. Fußnote 1), denen an dieser Stelle für ihr großes Engagement gedankt sei.

39 Für die UG wurden die Befragungsorte fakultätsweise festgelegt: Studierende der SOWI- sowie der REWI-Fakultät wurden jeweils am Haupteingang des Fakultätsgebäudes befragt, Studierende der THEOFakultät im Hörsaalbereich dieser Fakultät, Studierende der GEWIund der NAWI-Fakultät, die jeweils auf zahlreiche Gebäude aufgeteilt sind, an mehreren zentralen Orten (Haupteingänge einzelner Fakultätsgebäude bzw. Dekanate). An den übrigen Universitäten wurden die Befragungsorte universitätsweit festgelegt: An der TUG wurde an vier unterschiedlichen Orten (TU Bibliothek, Hauptgebäude der Alten Technik, Hauptgebäude der Neuen Technik, Studienzentrum), an der MUG sowie an der KUG an jeweils zwei verschiedenen Orten (MUG: Vorklinik und Zentrum für Medizinische Forschung beim LKH Graz; KUG: Hauptgebäude und „neues“ Gebäude) befragt, und zwar im jeweiligen zentralen Eingangsbereich.

40 Verteilung nach Fakultäten: GEWI: 364, NAWI: 247, SOWI: 249 REWI: 196, THEO: 25.

41 Aus der ersten Spalte in Tabelle 2 geht hervor, dass auch nicht alle der 1.931 für die Auswertung herangezogenen Fragebögen vollständig ausgefüllt sind. So fehlt z.B. in fünf Fragebögen die Angabe des Geschlechts $(n=1.926)$, in 13 Fällen fehlt die Angabe des Rauchverhaltens der Eltern $(\mathrm{n}=1.918)$.

42 Für die UG beträgt der Anteil der weiblichen Studierenden innerhalb der Stichprobe an der GEWI-Fakultät 81\%, an der NAWI- und der SOWI-Fakultät jeweils 54\%, an der REWI-Fakultät $43 \%$ und an der THEO-Fakultät $24 \%$.

43 Vgl. z.B. Biasco/Hartnett (2002).

44 Vgl. Metinta u.a. (1998).

45 Vgl. Kunze/Groman/Kunze (2004).

46 Beispielsweise durch die entgeltliche oder auch unentgeltliche Weitergabe von Zigaretten an unmündige Minderjährige.

47 Etwa durch das Tolerieren des Rauchens in Räumlichkeiten, die ihre Verantwortung unterliegen (z.B. Gastwirte tolerieren das Rauchen unmündiger Minderjähriger in ihren Lokalen oder Lehrer tolerieren das Rauchen von unmündigen Schülern in „ihrer“ Schule).

48 Vgl. Sundh/Hagquist (2005).

49 In den USA wird sogar über eine Anhebung des Schutzalters auf 21 Jahre nachgedacht (vgl. Ahmad (2005)).

50 Erläuterung zu den Altersklassen: „bis 13“ bedeutet von der Geburt bis einen Tag vor dem 14. Geburtstag, „14-15“ bedeutet vom 14. Geburtstag bis einen Tag vor dem 16. Geburtstag, usw.

51 Das Alter, mit dem in Österreich üblicherweise mit einem Studium begonnen wird, lässt sich bestenfalls als Zeitraum zwischen dem 18 . und dem 20. Lebensjahr angeben, da das Schuleintrittsalter je nach Geburtstag bei 6 oder 7 Jahren liegt, die Matura (Abitur) je nach Schultyp nach 12 oder 13 Schuljahren abgelegt wird und männliche Staatsbürger oftmals vor Studienbeginn ihren Wehr(ersatz)dienst ableisten.

52 Vgl. Ridner (2005).

53 Vgl. Pechmann/Reibling (2006).

54 Vgl. dazu den Beitrag von Wegner u.a. (2005) zu einer Abschätzung von direkten medizinischen Kosten als Folge tabakbedingter Erkrankungen für das Gesundheitswesen in Deutschland. Sopp (2005) hingegen stellt die Sinnhaftigkeit einer Erhöhung von Krankenversicherungsbeiträgen für Raucher in Frage.

55 Vgl. dazu auch Okuyemi u.a. (2001).

56 Erwartungsgemäß sind Gewichtsüberlegungen bei weiblichen Rauchern häufiger relevant als bei ihren männlichen Kommilitonen: Für 6\% der weiblichen, aber für nur 3\% der männlichen Raucher sind Gewichtsüberlegungen für das aktuelle Rauchverhalten von Bedeutung.

57 Vgl. Cawley/Markowitz/Tauras (2004).

58 Gleiches gilt hinsichtlich der nachfolgend behandelten Gründe für das Nichtrauchen.

59 Bezogen auf Raucher und Nichtraucher.

$60 \mathrm{Vgl}$. Haidinger/Waldhoer/Vutuc (1998).

61 Vgl. dazu auch Nicolas (2002).

62 Vgl. diesbezüglich auch Hammond u.a. (2007).

63 Eine fakultätsweise Betrachtung legt offen, dass der Anteil der Nichtraucher an der GEWI-Fakultät 64\%, an der SOWI-Fakultät 65\%, an der REWI-Fakultät 63\%, an der NAWI-Fakultät 61\% und an der THEO-Fakultät $84 \%$ (dabei ist allerdings zu berücksichtigen, dass an dieser Fakultät insgesamt nur 25 Studierende befragt wurden) beträgt.

64 Gewählte Altersgrenze: Vollendung des 22. Lebensjahres.

65 Wenn beide Elternteile rauchen, beträgt der Nichtraucheranteil nur mehr 46\%. 